\title{
SENSORS AND SYSTEMS FOR IN SITU OBSERVATIONS OF MARINE CARBON DIOXIDE SYSTEM VARIABLES
}

\author{
Robert H. Byrne ${ }^{(1)}$, Michael D. DeGrandpre ${ }^{(2)}$, R. Timothy Short ${ }^{(3)}$, Todd R. Martz ${ }^{(4)}$ Liliane Merlivat $^{(5)}$, \\ Craig McNeil $^{(6)}$, Fred L. Sayles ${ }^{(7)}$, Ryan Bell ${ }^{(3)}$, Peer Fietzek ${ }^{(9)}$ \\ ${ }^{(1)}$ College of Marine Science, University of South Florida, 140 7th Ave S, St. Petersburg, Florida, 33701 USA, \\ Email: byrne@marine.usf.edu \\ ${ }^{(2)}$ Department of Chemistry, University of Montana, Missoula, MT 59812-1296, USA \\ Email: michael.degrandpre@umontana.edu \\ ${ }^{(3)}$ SRI International (Stanford Research Institute), St. Petersburg, 450 8th Avenue SE St. Petersburg, FL 33701, \\ Email: timothy.short@sri.com; ryan.bell@sri.com \\ ${ }^{(4)}$ Scripps Institution of Oceanography, University of California San Diego, 9500 Gilman Drive, San Diego, La Jolla, \\ CA 92093-0225,USA, Email: trmartz@ucsd.edu \\ ${ }^{(5)}$ Université Pierre et Marie Curie, 4 place Jussieu 75005 Paris, France, Email: merlivat@locean-ipsl.upmc.fr \\ ${ }^{(6)}$ Applied Physics Laboratory, University of Washington, 1013 N.E. 40th St., Seattle, WA 98105, USA \\ Email: cmcneil@apl.washington.edu \\ ${ }^{(7)}$ Department of Chemistry and Geochemistry, Woods Hole Oceanographic Institution, Woods Hole, \\ 360 Woods Hole Rd., Clark, MS 25, Woods Hole, MA 02543, USA, Email: fsayles@whoi.edu \\ ${ }^{(8)}$ Leibniz Institute of Marine Sciences, Chemical Oceanography, University of Kiel, Düsternbrooker Weg 20, D-24105 \\ Kiel, Germany, Email: pfietzek@ifm-geomar.de
}

\begin{abstract}
Autonomous chemical sensors are required to document the marine carbon dioxide system's evolving response to anthropogenic $\mathrm{CO}_{2}$ inputs, as well as impacts on shortand long-term carbon cycling. Observations will be required over a wide range of spatial and temporal scales, and measurements will likely need to be maintained for decades. Measurable $\mathrm{CO}_{2}$ system variables currently include total dissolved inorganic carbon (DIC), total alkalinity $\left(\mathrm{A}_{\mathrm{T}}\right), \mathrm{CO}_{2}$ fugacity $\left(f_{\mathrm{CO} 2}\right)$, and $\mathrm{pH}$, with comprehensive characterization requiring measurement of at least two variables. These four parameters are amenable to in situ analysis, but sustained deployment remains a challenge. Available methods encompass a broad range of analytical techniques, including potentiometry, spectrophotometry, conductimetry, and mass spectrometry. Instrument capabilities (precision, accuracy, endurance, reliability, etc.) are diverse and will evolve substantially over the time that the marine $\mathrm{CO}_{2}$ system undergoes dramatic changes. Different suites of measurements/parameters will be appropriate for different sampling platforms and measurement objectives.
\end{abstract}

\section{STRATEGIES FOR OBTAINING COMPREHENSIVE CO $\mathrm{CO}_{2}$ SYSTEM CHARACTERIZATIONS}

Documentation of the marine carbon dioxide system's evolving response to anthropogenic $\mathrm{CO}_{2}$ inputs, as well as impacts on short- and long-term carbon cycling, will require sustained systematic and strategic measurement programs [1 and 2] that include the use of autonomous chemical sensors. Knowledge of two $\mathrm{CO}_{2}$ system parameters, including $\mathrm{CO}_{2}$ fugacity ${ }^{1}\left(f \mathrm{CO}_{2}\right)$, total alkalinity $\left(\mathrm{A}_{\mathrm{T}}\right)$, total dissolved inorganic carbon (DIC), $\mathrm{pH}$, and carbonate ion $\left(\mathrm{CO}_{3}{ }^{2-}\right)$ concentration, is sufficient for calculation of all system parameters. Shipboard methods for $f \mathrm{CO}_{2}, \mathrm{~A}_{\mathrm{T}}, \mathrm{DIC}$, and $\mathrm{pH}$ are well established. Field studies have shown that the accuracy and precision of calculated parameters depend upon an investigator's choice of thermodynamic constants and, more significantly, the choice of parameter-pairs [4]. Pair combinations that provide optimum results are $\mathrm{pH}-$ $\mathrm{A}_{\mathrm{T}}, \quad \mathrm{pH}-\mathrm{DIC}, f \mathrm{CO}_{2}-\mathrm{A}_{\mathrm{T}}$, or $f \mathrm{CO}_{2}-\mathrm{DIC}$. However, calculations involving $\mathrm{A}_{\mathrm{T}}$ require, in some cases, ancillary nutrient data. Measurement procedures for other parameters, e.g. $\mathrm{CO}_{3}{ }^{2-}$, are currently under development [5], and their contribution to uncertainty in $\mathrm{CO}_{2}$ system calculations has yet to be determined.

In the near term, $\mathrm{pH}$ or $f \mathrm{CO}_{2}$ sensors combined with sensors for $\mathrm{A}_{\mathrm{T}}$ or DIC offer the best approach for $\mathrm{CO}_{2}$ system characterization. Autonomous $\mathrm{fCO}_{2}$ and $\mathrm{pH}$ sensors are well established and commercially available [6, 7, 8 and 9] (Fig. 1). Most of these sensors utilize spectrophotometric measurements of $\mathrm{pH}$ in artificial test solutions of known alkalinity. Test solution $\mathrm{fCO}_{2}$ is calculated from $\mathrm{pH}$ and $\mathrm{A}_{\mathrm{T}}$ after the test solution equilibrates with seawater $\left(\left(f \mathrm{CO}_{2}\right)_{\text {test }}=\left(f \mathrm{CO}_{2}\right)_{\text {seawater }}\right)$ across a gas-permeable membrane. For $\mathrm{pH}$ measurements, seawater is directly mixed with a $\mathrm{pH}$ sensitive dye solution. In these types of sensors, the

\footnotetext{
${ }^{1}$ Atmospheric $\mathrm{CO}_{2}$ fugacity is numerically very similar to $\mathrm{CO}_{2}$ partial pressure. At equilibrium, it is the $\mathrm{CO}_{2}$ fugacities of seawater and the atmosphere $\left(f \mathrm{CO}_{2}\right)$ that are identical, not $\mathrm{CO}_{2}$ partial pressures $\left(p \mathrm{CO}_{2}\right)$. Fugacities are to partial pressures what activities are to concentrations [3].
} 
response is based on light absorption by an indicator dye at one or more wavelengths [6, 7, 8 and 9]. Measurement of $\mathrm{CO}_{2}$ by means of infrared absorption in an equilibrated seawater headspace is also common. Here equilibration can be achieved either by different equilibrator designs (e.g. a bubble-type equilibrator [7]) or by means of membranes in different forms [10]. Other types of $p \mathrm{CO}_{2}$ and $\mathrm{pH}$ instruments are in development [11, 12 and 13]. At present, there is a lack of available proven DIC and $\mathrm{A}_{\mathrm{T}}$ sensor technologies. Exceptional analytical performance is required for these measurements $(<0.1 \%$ precision and accuracy), and the shipboard techniques that have achieved this have done so through complex automated fluidic designs with frequent calibrations. Some groups have built prototype sensors for DIC [12 and 14] and $\mathrm{A}_{\mathrm{T}}$ [15] by using simplified detection methods. These systems are the most promising near-term prospects for full $\mathrm{CO}_{2}$ system characterization; however, they all require moving parts in the form of pumps and valves.

\section{MAINTENANCE OF IN SITU INSTRUMENTATION}

For a $\mathrm{CO}_{2}$ system ocean observing network to be successful, sufficient resources must be available for data quality validation and instrument maintenance. Taking the Argo program approach (www.argo.ucsd.edu ), a clear goal must be established and the most effective means for achieving this goal must be promoted. Assuming the goal is of sufficiently broad interest, the necessary monetary resources will follow. In developing the program, data validation will be crucial and the limitations of current instrumentation, such as long-term stability and biofouling resistance, must be accepted and dealt with in a realistic manner to ensure optimal quality data. Yearlong deployments are often promoted as a requirement, and in some cases this has been achieved [18 and 19], but deployment time frames should be dictated by the importance of the information being obtained and the performance of the
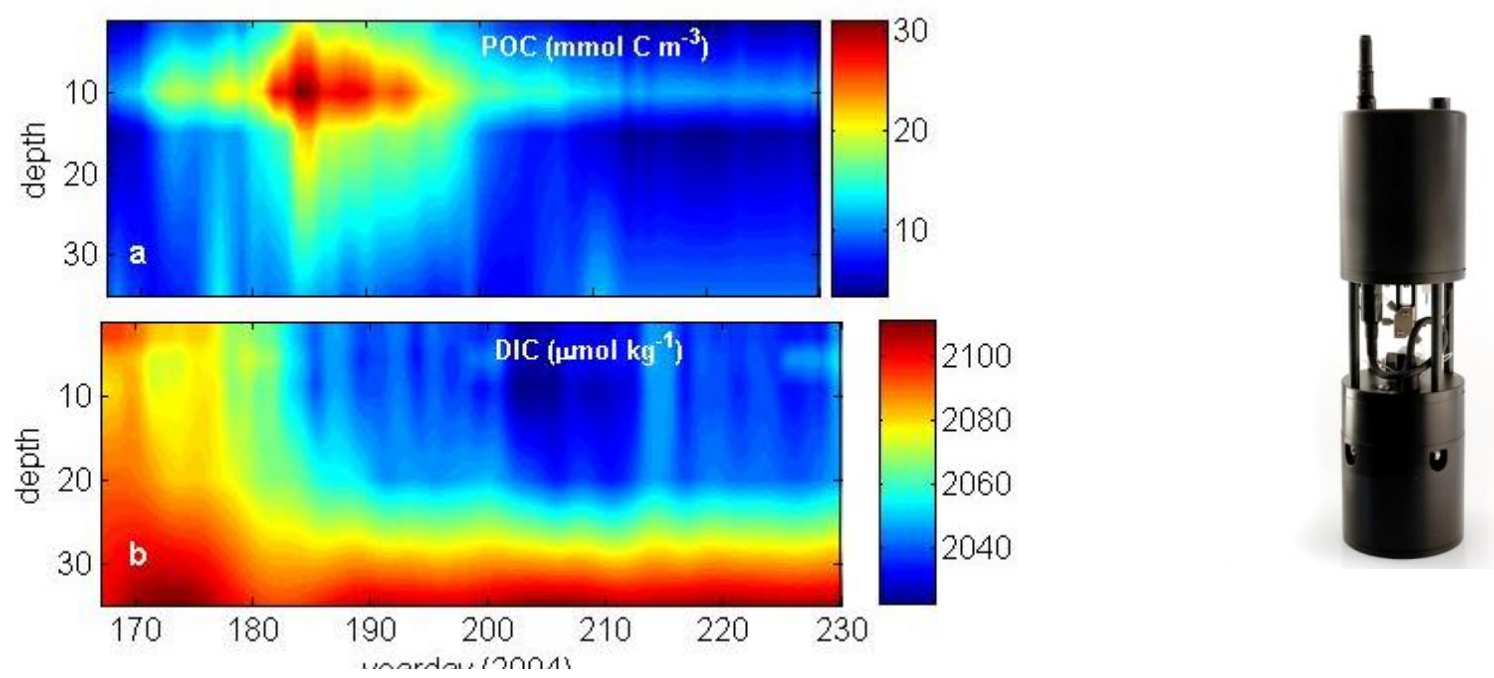

Figure 1. Particulate organic carbon (POC) and total dissolved inorganic carbon (DIC) time-series from a sensor string deployed in the Labrador Sea during the spring-summer bloom. DIC was calculated using in situ pCO $\mathrm{CO}_{2}$ measurements made with the $\mathrm{SAMI}-\mathrm{CO}_{2}$ (right) and alkalinity derived from temperature and salinity. The sensors captured the drawdown in DIC and increase in POC related to the increase in primary production as the mixed layer shoaled (taken from [17]).

In the long term, if $\mathrm{CO}_{2}$ system sensors are to be widely used, they need to evolve to reduce complexity and improve reliability. Oxygen sensors provide a good analogy - improvements in the amperometric $\mathrm{O}_{2}$ electrode [16] led to the first in situ $\mathrm{O}_{2}$ sensors, which spurred more research and interest from other instrument manufacturers, eventually resulting in development of the very stable $\mathrm{O}_{2}$ optode now available through a number of manufacturers. Development of the next generation of $\mathrm{CO}_{2}$ parameter sensors will require thinking outside the "box" of traditional methodologies used to characterize the $\mathrm{CO}_{2}$ system. sensors being utilized.

\section{APPROPRIATE PAIRING OF SENSORS AND MEASUREMENT PLATFORMS}

$\mathrm{CO}_{2}$ parameter sensors have been deployed on moorings, surface and Lagrangian drifters, and AUVs (Autonomous Underwater Vehicles). Power, size, and response time have made it problematic to use $\mathrm{CO}_{2}$ system sensors on existing Argo-type autonomous buoyancy-driven profilers and gliders. However, a 
recent field study has demonstrated the general feasibility of equipping profiling floats with $p \mathrm{CO} 2$ sensors [B. Fiedler, Leibniz Inst. Marine Sciences, Kiel, Germany, pers. comm.]. Larger-capacity profilers can also be readily designed for a wider range of sensor types. Secondly, profiling routines can be altered from the traditional Argo approach, i.e., profilers can be programmed to move more slowly for slowresponding sensors and profile only the surface ocean (e.g. < $200 \mathrm{~m})$. Some profilers have already demonstrated such capabilities [20]. As stated above, simplified sensors without pumps, valves, and other moving parts need to be developed, akin to the $\mathrm{O}_{2}$ optode, to enable widespread use of $\mathrm{CO}_{2}$ system sensors on profilers and gliders.

Current $\mathrm{CO}_{2}$ system sensor technologies can readily be deployed on fixed platforms or at variable depths via winched profilers, as proposed by the U.S. Ocean Observatory Initiative (OOI, www.oceanleadership.org/ocean_observing). Power requirements are limiting in most but not all deployment scenarios. Autonomous profilers and gliders have very limited payloads that prohibit large power sources. New autonomous surface vehicles and modified $\mathrm{CO}_{2}$ sensors have been proposed to address these problems [e.g. 21] Other platforms, such as the moorings being designed as part of the U.S. Ocean Observatory Initiative [22], will have sufficient power that deployment durations can be extended to $\sim 1$-year periods for a variety of advanced instrumentation. In this case, in situ calibration capabilities and biofouling resistance would become performance-limiting factors, rather than battery endurance. In addition, response time is generally not an issue at fixed locations and depths, and winched profilers can be programmed to dwell at fixed depths, consistent with sensor response times.

Isopycnal sampling offers an additional alternative to rapidly profiling platforms, gliders, and fixed moorings [23]. Isopycnal sampling effectively removes the complicating effects of internal waves typical of moored data. Isopycnal sampling provides the opportunity to fully thermally equilibrate slow-response sensors prior to measurement-taking. Though modern electronics usually provide good temperature compensation, the physical behavior of some sensing components (e.g., optical alignments) may require extensive equilibration and/or temperature calibration. Autonomous profiling floats can provide isopycnal sensing through CTD (Conductivity-Temperature-Depth) input to a float's buoyancy control engine.

\section{INTEGRATION OF EMERGING AUTONOMOUS TECHNOLOGIES WITH EXISTING ANALYTICAL PROTOCOLS}

Two ingredients are necessary for acceptance of new technology. First, new sensors must be extensively tested side-by-side with conventional methods (e.g., the Technology Demonstration projects of the Alliance for Coastal Technologies, www.act-us.info). Initially, such tests are probably best performed shipboard, where a wide range of constituent concentrations, temperatures, and salinities will be encountered. Subsequently, test sites need to be chosen and populated with sensors for long-term comparisons of established and developing technologies. Secondly, and quite importantly, in situ technologies should be commercialized so that they are available to the oceanographic community for utilization and testing under the widest possible range of circumstances. Sensors can be considered to be truly robust only when they perform well in the hands of nonexperts. If a technology proves reliable and costeffective, it will be quickly adopted by researchers, as has been the case with $\mathrm{O}_{2}$ optode technology.

\section{SENSOR CALIBRATION AND ENHANCEMENT OF MEASUREMENT ACCURACY}

The ability to perform in situ quality control, either in the form of calibrations or metadata analysis, will be critical for evaluating sensor performance and establishing measurement uncertainty. It is noted, however, that in situ calibration contravenes simplicity because introduction of a standard or certified reference material (CRM) requires an additional pump or valve, or both. Standards or CRMs [24 and 25] can be used for $\mathrm{pH}$, DIC, or $\mathrm{A}_{\mathrm{T}}$, but there is no practical in situ standardization method for $f \mathrm{CO}_{2}$ sensors. Even infraredbased in situ sensors (e.g. [7]) calibrate only the infrared analyzer and not the equilibration process that, by itself, can contribute significantly to analytical errors [26]. For facile use of CRMs in situ, there is a vital need to extend Andrew Dickson's CRM program whereby CRMs are distributed in durable, gas-impermeable bags suitable for deployment in situ. The authors of [14] have shown that DIC levels are stable for long periods when CRMs are stored in polyethylene-coated aluminum bags (Fig. 2). Systematic evaluation of reagent stability should be included as an integral part of the use of in situ standards for calibrations. Additionally, in cases where in situ calibrations are impractical, system metadata may be used to identify suspect data. For 
example, in indicator-based $\mathrm{CO}_{2}$ and $\mathrm{pH}$ sensors, absorbance values may be used to flag data that fall outside an accepted range.

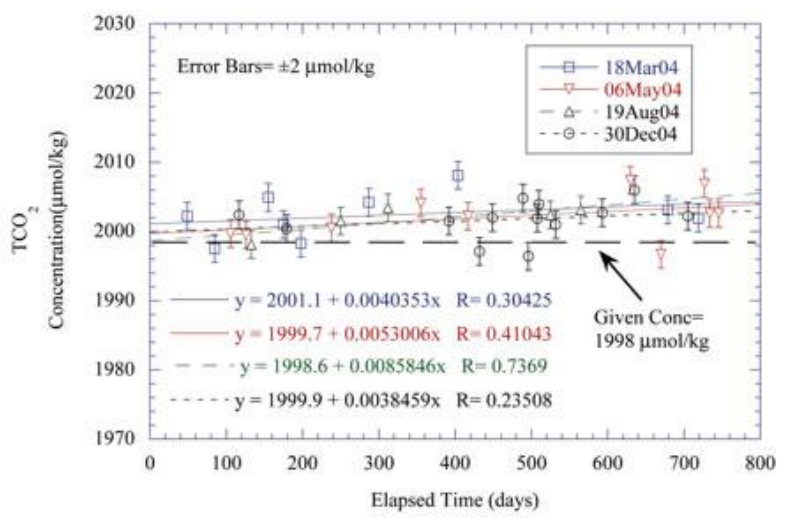

Figure 2. The change in certified reference material

(CRM) DIC over time when the CRM is stored in

polyethylene-coated Al bags. Cali-5-Bond ${ }^{\mathrm{TM}}$ bags were rinsed and filled with $500 \mathrm{cc}$ of CRM Batch 61 and periodically analyzed for DIC. The drift corresponds to $\sim 2 \mathrm{\mu mol} \mathrm{kg}^{-1} \mathrm{yr}^{-1}$ (from [14]).

It may be feasible to use historical $\mathrm{CO}_{2}$ measurements in deep water (e.g. $\geq \sim 2000 \mathrm{~m}$ ) to improve the quality of $\mathrm{CO}_{2}$ system measurements obtained from profiling floats or benthic landers. Similar methods are currently being implemented for Argo salinity measurements [27]. However, as shown for recent $\mathrm{O}_{2}$ data obtained from profiling floats [28], poor data coverage will limit this approach in many areas of the ocean. Additionally, single point calibrations in air when floats surface may be useful for $p \mathrm{CO}_{2}$ sensors, in a manner similar to techniques now being evaluated for oxygen sensors (A. Körtzinger, Leibniz Inst. Marine Sciences, Kiel, Germany, pers. comm.).

Redundant sensors should be deployed whenever possible as an additional check on sensor performance. Although it is difficult to determine which record is accurate when two sensors develop divergent outputs, concordance of sensor records greatly enhances confidence in the quality of sensor data. In some cases, two different technologies that measure the same parameter can be combined to advantage. A particular type of sensor may be more versatile but less stable than another type of sensor measuring the same parameter. For example, an "industrial production of integrated ion sensitive field effect transistor" (ISFET) pH sensor (13] deployed on a glider could return to a central base where an in situ spectrophotometric sensor (e.g. [9]) verifies data quality. It may also prove useful to develop some sensor/platform combinations that serve as calibration systems for an extended suite of fixed-site sensors. When the spatial distribution of sensor arrays becomes sufficiently dense, it may be effective to use high-quality glider-borne sensors, with brief but frequent deployment cycles, to calibrate fixed-location sensors that remain in place over long periods of time.

\section{USE OF SYSTEM THERMODYNAMICS TO ASSESS AND PROMOTE DATA QUALITY}

It is clearly vital that thermodynamic models be used to assess in situ $\mathrm{CO}_{2}$ data sets. Shipboard studies have added importantly to assessments of model accuracy. Nevertheless, additional improvements are essential. Studies are initially best undertaken on ships where the best quality measurements can be made. However, through time, in situ data will come to dwarf the quantity of shipboard data. As such, it is essential to make good use of in situ data for thermodynamic consistency evaluations. This might begin with redetermination of the stability constants $K_{l}$ and $K_{2}$ over the full range of oceanic temperature, salinity, and pressure.

Good use should also be made of the relatively conservative behavior of $\mathrm{A}_{\mathrm{T}}$. Regional characterizations of relationships between $\mathrm{A}_{\mathrm{T}}$, salinity, and temperature [29 and 30] will allow integration of directly measured and calculated alkalinity when sensors are deployed in areas where relationships among these three quantities are slowly varying functions of latitude and longitude (e.g. Fig. 1). Thus, whether directly measured or calculated, $\mathrm{A}_{\mathrm{T}}$ can serve as an especially useful integrating component in evaluations of the internal consistency of field measurements, rather than serving simply as one of four possible components in thermodynamic consistency evaluations.

\section{INTRODUCTION OF NEW TECHNOLOGIES}

A number of additional technologies are being developed to quantify $\mathrm{CO}_{2}$ system parameters. Portable underwater membrane inlet mass spectrometry (MIMS) systems offer the potential for in situ characterizations of the carbon system using a single instrument by simultaneous determination of $\mathrm{fCO}_{2}$ and DIC. In situ MIMS systems have been used to determine vertical dissolved gas concentrations of light stable gases at unprecedented spatial resolution [31] and have been deployed on a variety of platforms [32, 33 and 34]. With proper calibration, $f \mathrm{CO}_{2}$ can be determined using in situ MIMS by direct measurement of dissolved gaseous $\mathrm{CO}_{2}$. In situ acidification of seawater should also allow use of MIMS for measurements of DIC. The capability to determine $f \mathrm{CO}_{2}$ and DIC using a single in situ instrument that also provides measurements of light stable dissolved gases (e.g., methane, oxygen, nitrogen, and argon) should offer important new opportunities for in situ carbon system measurements (Fig. 3). An additional advantage of MIMS measurements is the possibility of obtaining in situ carbon isotope measurements to constrain $\mathrm{CO}_{2}$ sources and assess the extent of biological fractionation, bubble injection, and 
other such processes. There is no doubt that this and other future innovations in chemical sensing technology will further enhance our ability to understand the ocean carbon cycle and $\mathrm{CO}_{2}$ sources and sinks.

\section{SUMMARY COMMENTS ON GOALS, CURRENT STATUS, AND FUTURE NEEDS}

The ocean can be viewed as a harsh environment for sensor deployments. However, in some respects, in situ conditions are relatively benign. As one example, even at shallow depths, polar waters are nearly isothermal and relatively quiescent compared to the "noisy" electro-mechanical and thermal environments on ships.
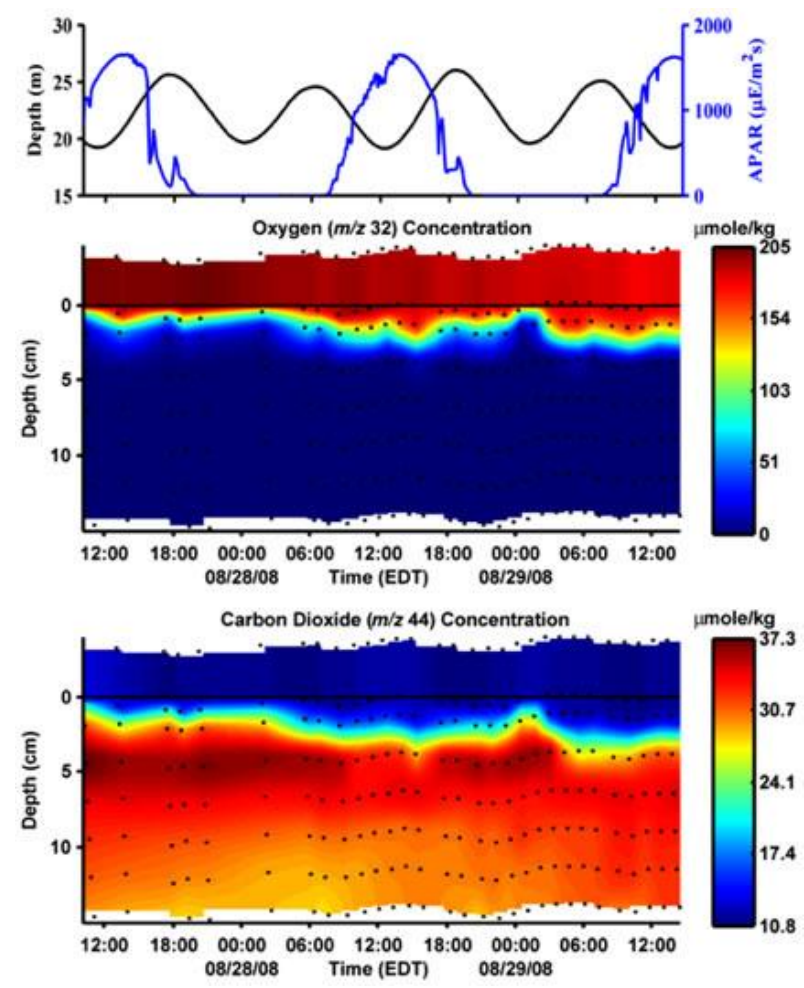

Figure 3. Oxygen and carbon dioxide concentrations in South Atlantic Bight sediment pore waters obtained with a MIMS instrument. A positive displacement syringe pump and a sediment probe were used to examine $\mathrm{O}_{2}$ and $\mathrm{CO}_{2}$ concentrations above and below the sediment/water interface. Variations in hydrostatic pressure and photosynthetically active radiation are also shown for the two-day observational period.

As another example, sensors deployed at the air/sea interface are exposed to challenging environmental extremes, but problems can be mitigated by positioning sensors within the mixed layer, removed from diurnal air-temperature variations. As such, despite unique problems such as biofouling that are inherent to in situ measurements, it is reasonable to anticipate that in situ instruments can eventually provide precisions and accuracies commensurate with shipboard measurements. Achievable goals for in situ measurement precision should be considered as $\sim 1$ $\mu \mathrm{mol} / \mathrm{kg}$ for DIC and $\mathrm{A}_{\mathrm{T}}, 0.0005$ to 0.001 for $\mathrm{pH}$, and $1 \mu \mathrm{atm}$ for $\mathrm{CO}_{2}$ fugacity. Acceptable accuracies for DIC and $\mathrm{A}_{\mathrm{T}}$ are likely to be closely coupled to in situ use of $\mathrm{CRMs}$, as is currently required for discrete shipboard measurements. Therefore, a critical enabling technology, one that should receive increasing emphasis, is the development of stable CRMs for DIC and $\mathrm{A}_{\mathrm{T}}$ measurements in situ. It should be noted, in contrast, that CRMs are not necessarily essential to the accuracy of $\mathrm{pH}$ measurements. The indicators used in spectrophotometric measurements are themselves molecular standards [35]. In this case, however, it is important that the indicators be pure [36] and that the linearity of the in situ spectrophotometric sensors be demonstrated via comparisons with high-quality benchtop spectrophotometers (e.g. [11]). For pH sensors that do not exhibit consistent linear photometric behavior, use of in situ CRMs is essential for quality assurance. CRMs do not currently exist for measurements of $\mathrm{CO}_{2}$ fugacity. As noted in the preceding discussion, however, calibrations can be obtained in some cases through measurements of atmospheric $p \mathrm{CO}_{2}$. Because assessments of precision and accuracy are critical, increasing efforts should be made to deploy compatible sensor pairs (DIC plus either $f_{\mathrm{CO} 2}$ or $\mathrm{pH}$, and/or $\mathrm{A}_{\mathrm{T}}$ plus either $f_{\mathrm{CO} 2}$ or $\mathrm{pH}$ ). Such judicious pairing will allow assessment of the overall quality and sustainability of comprehensive thermodynamic characterizations (calculations of four primary measured variables plus carbonate saturation state).

In the short term, it appears reasonable to emphasize deployment of compatible sensors on moorings within the mixed layer. Adapting sensors for deployment on gliders and floats is highly desirable too, but in view of the disparate response times of various sensors and size and power constraints, an initial emphasis on deployment at fixed depths and locations is likely to more readily yield comprehensive suites of quality measurements that can be used to validate sensor performance. Under conditions that change relatively slowly, equilibration can be assured, and measured and calculated parameters should exhibit simple, thermodynamically coupled variations through time, allowing for rigorous assessment of precisions and accuracies.

Relatively little work has been devoted to direct comparison of in situ $\mathrm{CO}_{2}$ system measurements with contemporaneous discrete measurements obtained using conventional shipboard procedures. Such comparisons are quite important and should receive increasing emphasis. The thermodynamic consistency and quality of shipboard carbon system measurements have been examined extensively via many ocean expeditions, so 
that comparisons against these standard measurements serve as essential benchmarks of progress toward achievable goals.

As one means of assessing in situ precision and accuracy for $\mathrm{pH}$, the only measured parameter with negligible equilibration times, it is reasonable to emphasize deployments with CTD-rosette systems for real-time observations. In situ $\mathrm{pH}$ profiles with 1-meter resolution or better, such as those obtained during the 2007 U.S. National Oceanic and Atmospheric Administration (NOAA) Gulf of Mexico and East Coast Carbon Cruise (GOMECC) field expedition (Fig. 4), allow excellent assessment of measurement precision.

GOMECC Station 79, July 31, 2007 (39'54.334N and 69'55.799W)
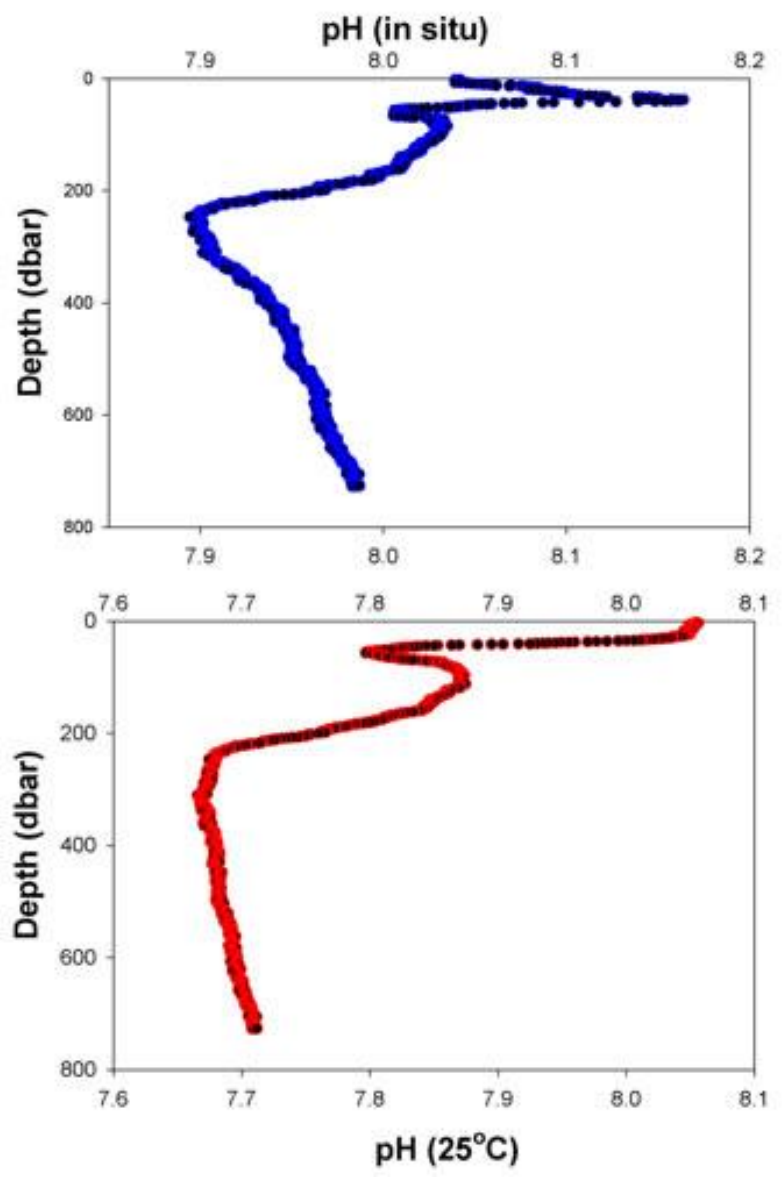

Figure 4. In situ pH measurements obtained during the 2007 NOAA GOMECC expedition. A

Spectrophotometric Elemental Analysis System (SEAS) II sensor replaced a single Niskin bottle on the rosette sampling frame and measured $\mathrm{pH}$ with a sampling frequency of approximately $1 \mathrm{~Hz}$. All measurements, including $\mathrm{pH}$, salinity, temperature, and depth, were stored in SEAS II FLASH memory and were also transmitted in real time to the research vessel.
Comparisons with observations of discrete Niskin samples can then provide insightful assessments of in situ accuracy.

Current availability of commercial and custom in situ sensors (see www.IOCCP.org) is limited to $\mathrm{CO}_{2}$ partial pressure and $\mathrm{pH}$. CARIOCA and Sunburst Sensors have exhibited excellent endurance (months to $\sim 1$ year) and a data quality sufficient for useful estimates of air/sea $\mathrm{CO}_{2}$ fluxes. The reported current precisions of in situ $\mathrm{pH}$ measurements obtained by the Sunburst Sensors are on the order of \pm 0.001 . This precision is quite acceptable for comprehensive $\mathrm{CO}_{2}$ system calculations. Further development of in situ DIC measurement capabilities is of great importance. As such, it would be quite useful to deploy the conductimetric DIC system of [14] in concert with deployments of existing $\mathrm{pH}$ and $f \mathrm{CO}_{2}$ sensors.

Such simultaneous deployments would allow a robust examination of thermodynamic consistency, measurement precision, and accuracy. To further the objective of comprehensive in situ $\mathrm{CO}_{2}$ system characterizations, it is also important to examine the efficacy of in situ DIC measurements obtained using alternative measurement protocols such as membrane introduction mass spectrometry and spectrophotometry. The semi-autonomous spectrophotometric DIC measurement system of [12] can be easily adapted for in situ analysis, and the MIMS system of [31] is well suited to in situ DIC measurements. Both of these technologies are currently poised for commercialization.

There are at least two principal impediments to widespread utilization of in situ instrumentation: cost and complexity. Developers of in situ instrumentation endeavor to reproduce the best qualities of laboratory analyses-but outside the benign laboratory environment and without the benefit of a hands-on analyst. While, from one perspective, in situ analysis can do much to reduce labor and its associated costs, in the absence of guaranteed data quality and prospects for simple maintenance, up-front capital expenditures can appear formidable. Much of the instrumentation that is currently commercial, or available for commercialization, requires expert, insightful care. In order for in situ instrumentation to realize its full potential for contributing to environmental analysis, the instrumentation must be widely utilized. Extensive utilization will, in turn, reduce per-unit costs and, importantly, provide the critical assessments of system performance that are essential to evolution of instrument design. Thus, widespread sensor utilization, low cost, and improved performance are inextricably linked.

\section{REFERENCES}

1. Feely, R. \& Co-Authors (2010). "An International Observational Network for Ocean Acidification" in these proceedings (Vol. 2), doi:10.5270/OceanObs09.cwp.29. 
2. Monteiro, P. \& Co-Authors (2010). "A Global Sea Surface Carbon Observing System: Assessment of Changing Sea Surface $\mathrm{CO} 2$ and Air-Sea CO2 Fluxes" in these proceedings (Vol. 2), doi:10.5270/OceanObs09.cwp.64.

3. Morel, F.M.M. \& Hering, J.G. (1993) Principles and Applications of Aquatic Chemistry, John Wiley \& Sons, New York, NY, USA, p. 79.

4. Millero, F.J. (2007) The Marine Inorganic Carbon Cycle. Chem. Rev. 107, 308 - 341.

5. Byrne, R.H. \& Yao, W. (2008) Procedures for Measurement of Carbonate Ion Concentrations in Seawater by Direct Spectrophotometric Observations of $\mathrm{Pb}$ (II) Complexation. Mar. Chem. 112, 128-135.

6. DeGrandpre, M.D., Hammar, T.R., Smith, S.P. \& Sayles, F.L. (1995). In Situ Measurements of Seawater $p \mathrm{CO}_{2}$. Limnol. Oceanog. 40, 969-975.

7. Friederich, G.E., Brewer, P.G., Herlien, R. \& Chavez, F.P. (1995) Measurement of Sea Surface Partial Pressure of $\mathrm{CO}_{2}$ from a Moored Buoy. Deep-Sea Res. I 42, 11751186.

8. Lefèvre, N., Ciabrini, J.P., Michard, G., Brient, B., DuChaffaut, M. \& Merlivat, L. (1993) A New Optical Sensor for $p \mathrm{CO}_{2}$ Measurements in Seawater. Mar. Chem. 42, 189-198.

9. Seidel, M.P., DeGrandpre, M.D. \& Dickson, A.G. (2008) A Sensor for In Situ Indicator-Based Measurements of Seawater pH. Mar. Chem. 109, 18-28.

10. Fietzek, P. \& Körtzinger, A. (2010). "Optimization of a Membrane Based NDIR-sensor for Dissolved CO2" in these proceedings (Annex), doi:10.5270/OceanObs09.

11. Liu, X., Wang, Z., Byrne, R.H., Kaltenbacher, E.A. \& Bernstein, R.E. (2006) Spectrophotometric Measurements of pH In Situ: Laboratory and Field Evaluations of Instrumental Performance. Environ. Sci. Technol. 40, 5036-5044.

12. Wang, Z.A., Liu, X., Byrne, R.H., Wanninkhof, R., Bernstein, R., Kaltenbacher, E. \& Patten, J. (2007) Simultaneous Spectrophotometric Flow-Through Measurements of pH, Carbon Dioxide Fugacity, and Total Inorganic Carbon in Seawater. Anal. Chim. Acta 596, 23-36.

13. Martz, T. R., J. G. Connery and K. S. Johnson. 2010. Testing the Honeywell Durafet ${ }^{\circledR}$ for seawater $\mathrm{pH}$ applications. Limnology and Oceanography: Methods, $8,172-184$

14. Sayles, F.L. \& Eck, C.F. (2009) An autonomous Instrument for Time Series Analysis of $\mathrm{TCO}_{2}$ from Oceanographic Moorings. Deep-Sea Res. Part I 56(9), 1590-1603.

15. Martz, T.R., Dickson, A.G. \& DeGrandpre, M.D. (2006) Tracer Monitored Titrations: Measurement of Total Alkalinity. Anal. Chem. 78, 1817-1826.

16. Langdon, C. (1984) Dissolved Oxygen Monitoring System Using a Pulsed Electrode: Design, Performance, and Evaluation. Deep-Sea Res. I 31, 1357-1367.
17. Martz, T.M., DeGrandpre, M.D., Strutton, P.G., McGillis, W.R. \& Drennan, W. (2009) Sea Surface $p \mathrm{CO}_{2}$ and Carbon Export during the Labrador Sea Spring-Summer Bloom: An In Situ Mass Balance Approach. J. Geophys. Res. - Oceans 114(C09008), doi:10.1029/2008JC005060.

18. Boutin J., Merlivat, L., Hénocq, C., Martin, N. \& Sallée, J.B. (2008) Air-Sea $\mathrm{CO}_{2}$ Flux Variability in Frontal Regions of the Southern Ocean from CARIOCA Drifters. Limnol. Oceanogr. 53, 2062-2079.

19. DeGrandpre, M.D., Körtzinger, A., Send, U., Wallace, D.W.R. \& Bellerby, R.G.J. (2006) Uptake and sequestration of atmospheric $\mathrm{CO}_{2}$ in the Labrador Sea deep convection region. Geophys. Res. Lett. 33, doi:10.1029/2006GL026881.

20. Bishop, J.K.B., Wood, T.J., Davis, R.E. \& Sherman, J.T. (2004) Robotic Observations of Enhanced Carbon Biomass and Export at 55S. Science 304, 417-420.

21. Richardson, T. \& Co-Authors (2010). "An Autonomous Mobile Platform for Underway Surface Carbon Measurements in Open-Ocean and Coastal Waters" in these proceedings (Annex), doi:10.5270/OceanObs09.

22. Brasseur, L., Tamburri, M. and Pluedemann, A., (2010). "Sensor Needs and Readiness Levels for Ocean Observing: An Example from the Ocean Observatories Initiative (OOI)" in these proceedings (Vol. 2), doi:10.5270/OceanObs09.cwp.10.

23. D'Asaro, E.A. \& McNeil, C.L. (2007) Air-Sea Gas Exchange at Extreme Wind Speeds Measured by Autonomous Oceanographic Floats. J. Mar. Syst. 66, 92-109.

24. Nemzer, B.V. \& Dickson, A.G. (2005) The Stability and Reproducibility of Tris Buffers in Synthetic Seawater. Mar. Chem. 96, 237-243.

25. Dickson, A.G., J.D. Afghan \& Anderson, G.C. (2003) Reference Materials for Oceanic $\mathrm{CO}_{2}$ Analysis: A Method for the Certification of Total Alkalinity. Mar. Chem. 80, 185-197.

26. Körtzinger, A., Mintrop, L., Wallace, D.W.R., Johnson, K.M., Neill, C., Tilbrook, B., Towler, P., Inoue, H.Y., Ishii, M., Shaffer, G., Saavedra, R.F., Ohtaki, E., Yamashita, E., Poisson, A., Brunet, C., Schauer, B., Goyet, C. \& Eischeid, G. (2000) The International At-Sea Intercomparison of $f \mathrm{CO}_{2}$ Systems during the R/V Meteor Cruise 36/1 in the North Atlantic Ocean. Mar. Chem. 72, 171-192.

27. Wong, A.P.S., Johnson, G.C. \& Owens, W.B. (2003) Delayed-Mode Calibration of Autonomous CTD Profiling Float Salinity Data by $\theta-S$ Climatology. J. of Atm. Ocean. Tech. 20, 308-318.

28. Martz, T. R., Johnson, K.S. \& Riser, S.C. (2008) Ocean Metabolism Observed with Oxygen Sensors on Profiling Floats in the South Pacific. Limnol. Oceanogr. 53, 2094 2111.

29. Lee, K., Tong, L., Millero, F., Sabine, C., Dickson, A., Goyet, C., Park, G., Wanninkhof, R., Feely, R. \& Key, R. (2006) Global Relationship of Total Alkalinity and 
Temperature in Surface Waters of the World's Oceans. Geophys. Res. Lett. 33, L19605/1-L19605/5.

30. Merlivat, L., Gonzalez-Davila, M., Caniaux, G., Boutin, J. \& Reverdin, G. (2009) Mesoscale and Diel to Monthly Variability of $\mathrm{CO}_{2}$ and Carbon Fluxes at the Ocean Surface in the Northeastern Atlantic. J. Geophys. Res. 114, C03010, doi:10.1029/2007JC004657.

31. Bell, R.J., Short, R.T., van Amerom, F.H.W. \& Byrne, R.H. (2007) Calibration of an In Situ Membrane Inlet Mass Spectrometer for Measurements of Dissolved Gases and Volatile Organics in Seawater. Environ. Sci. Technol. 41 8123-8128.

32. Wenner, P.G., Bell, R.J., van Amerom, F.H.W., Toler, S.K., Edkins, J.E., Hall, M.L., Koehn, K., Short, R.T. \& Byrne, R.H. (2004) Environmental Chemical Mapping Using an Underwater Mass Spectrometer. Trends Anal. Chem.: Special Issue on Deploying Mass Spectrometers in Harsh Environments 23, 288-295.

33. Camilli, R. \& Hemond, H.F. (2004) NEREUS/Kemonaut, a Mobile Autonomous Underwater Mass Spectrometer. Trends Anal. Chem. 23, 307-313.

34. Schlueter, M. \& Gentz, T. (2008) Application of Membrane Inlet Mass Spectrometry for Online and In Situ Analysis of Methane in Aquatic Environments. $J$. Am. Soc. Mass Spectrom. 19, 1395-1402.

35. Byrne, R.H. (1987) Standardization of Standard Buffers by Visible Spectrometry. Anal. Chem. 59, 1479-1481.

36. Yao, W., Liu, X. \& Byrne, R.H. (2007) Impurities in Indicators Used for Spectrophotometric Seawater $\mathrm{pH}$ Measurements: Assessment and Remedies. Mar. Chem. 107, 167-172. 